\title{
Eosinophilic Lung Disease Complicated by Kimura's Disease: A Case Report and Literature Review
}

\author{
Hidefumi Koh ${ }^{1}$, Nobufumi Kamiishi ${ }^{1}$, Atsushi Chiyotani ${ }^{1}$, Hidenori Takahashi ${ }^{1}$, \\ Akihiko Sudo ${ }^{1}$, Yoshihiro Masuda ${ }^{2}$, Seiichi Shinden ${ }^{3}$, Atsushi Tajima ${ }^{4}$, \\ Yoshishige Kimura ${ }^{4}$ and Tokuhiro Kimura ${ }^{5}$
}

\begin{abstract}
Kimura's disease (KD) or eosinophilic lymphogranuloma is a rare chronic inflammatory disorder of unknown etiology that occurs primarily in Asians. A 51-year-old man diagnosed three years earlier with KD of a left neck nodule was admitted to our hospital with a productive cough and pulmonary infiltration. Bronchoscopy was performed, and a diagnosis of eosinophilic lung disease (ELD) was made. The patient's condition improved after receiving corticosteroid treatment. Complications such as nephrotic syndrome have been reported in patients with KD; however, ELD has not been previously described. To the best of our knowledge, this is the first report of ELD related to KD.
\end{abstract}

Key words: Kimura's disease, eosinophilic lymphogranuloma, eosinophilic lung disease

(Intern Med 51: 3163-3167, 2012)

(DOI: 10.2169/internalmedicine.51.8600)

\section{Introduction}

Kimura's disease (KD) was first described in 1948 by Kimura and coworkers (1). The disease is a rare chronic inflammatory disorder of unknown etiology that occurs primarily in Asians. Elevated serum immunoglobulin E ( $\mathrm{IgE}$ ) levels and peripheral blood eosinophilia are commonly seen in patients with this condition. Allergic reactions, infections and autoimmune reactions, including aberrant immune reactions, have been suggested as causes; nevertheless, the etiology and pathogenesis remain unknown. In a review of 429 Japanese cases of KD, Ishii identified associations in 14 cases of nephritic syndrome, five cases of bronchial asthma, three cases of allergic rhinitis, two cases of atopic dermatitis and five cases of urticaria, suggesting that an allergic mechanism may have contributed to $\mathrm{KD}$ in these patients (2). No associations between KD and diffuse lung disorders such as eosinophilic lung disease (ELD) have been previously described. We herein report a case of ELD complicated by KD.

\section{Case Report}

A 51-year-old man was admitted to the medical unit of our hospital in December 2009 with a productive cough and dyspnea. Two weeks prior to admission, he had experienced a productive cough, fever and dyspnea. One week prior to admission, he was seen at the emergency department of another hospital and diagnosed with community acquired pneumonia. After one week of antibiotic therapy, no improvement was seen in his condition, and he was subsequently referred to our hospital for further evaluation and treatment.

The patient had been admitted to our hospital three years prior with a left neck nodule, blood eosinophilia (13\%) and a high IgE level $(351 \mathrm{mg} / \mathrm{dL}$, normal range: <173). The nodule was indurative, mobile, non-tender and measured ap-

\footnotetext{
${ }^{1}$ Division of Pulmonary Medicine, Department of Internal Medicine, Saiseikai Utsunomiya Hospital, Japan, ${ }^{2}$ Division of Hematology, Department of Internal Medicine, Saiseikai Utsunomiya Hospital, Japan, ${ }^{3}$ Department of Otorhinolaryngology, Head \& Neck Surgery, Saiseikai Utsunomiya Hospital, Japan, ${ }^{4}$ Department of General Thoracic Surgery, Saiseikai Utsunomiya Hospital, Japan and ${ }^{5}$ Department of Pathology, Keio University School of Medicine, Japan

Received for publication July 13, 2012; Accepted for publication August 21, 2012
}

Correspondence to Dr. Hidefumi Koh, hdfmkoh@yahoo.com 

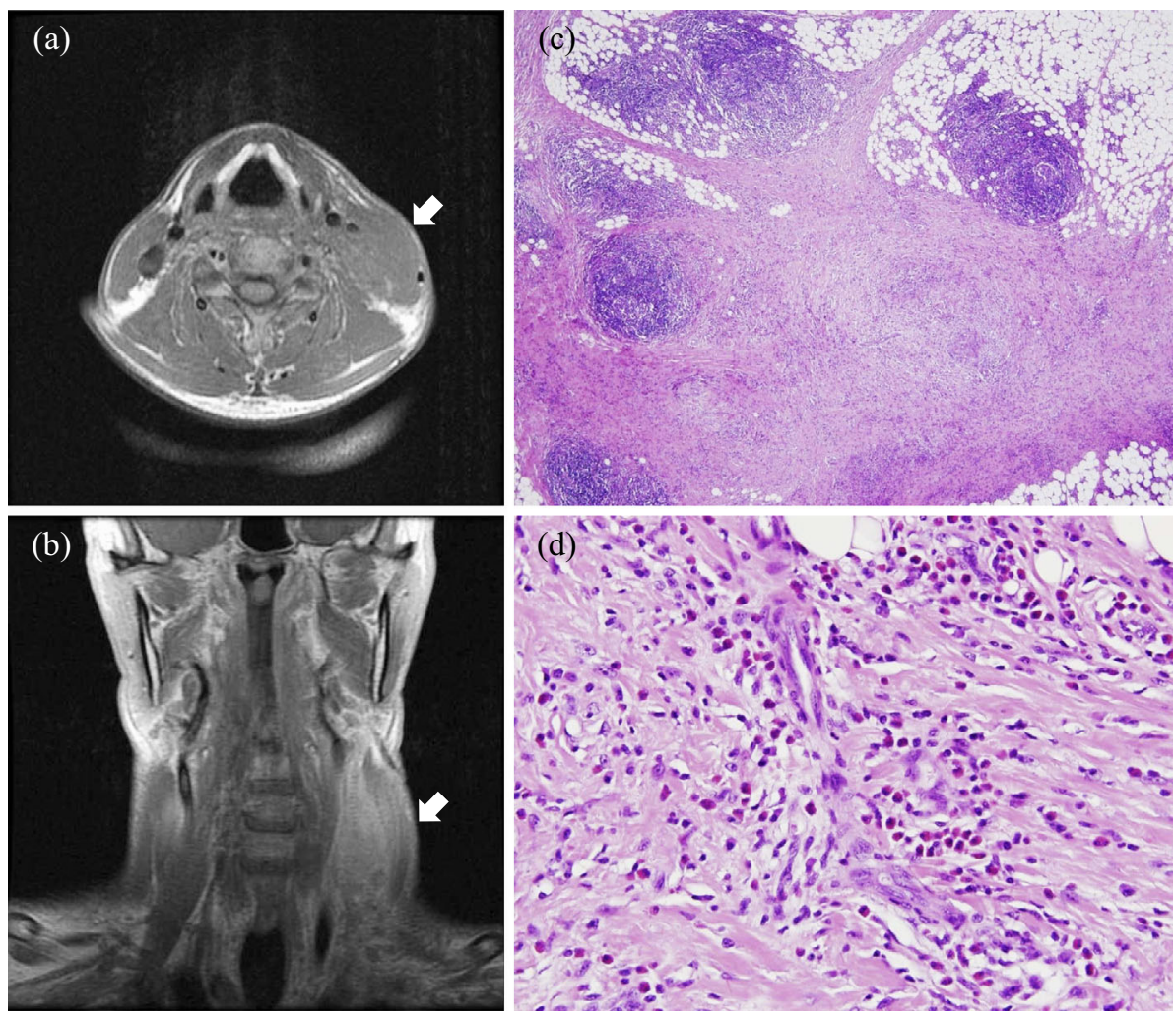

Figure 1. a, b: Magnetic resonance imaging demonstrating a low intensity mass in the left neck (arrow). c, d: Microscopic findings of the resected specimen revealing lymphoid hyperplasia, eosinophil infiltration, fibrosis and small blood vessels (Hematoxylin and Eosin staining; original magnification: $\mathrm{c}, \times 100 ; \mathrm{d}, \times \mathbf{4 0 0})$.

proximately $6 \times 2.5 \mathrm{~cm}$. Magnetic resonance imaging $(\mathrm{MRI})$ revealed a low intensity mass in the left neck (Fig. 1a, b). An excision biopsy was performed and a histological examination of the specimen revealed lymphoid hyperplasia, eosinophil infiltration, fibrosis and small blood vessels (Fig. 1c, d). The patient was diagnosed with $\mathrm{KD}$ and received medical follow-up with no signs of recurrence. However, the blood eosinophil level ranged from $4 \%$ to $14 \%$ and the IgE level ranged from $283 \mathrm{mg} / \mathrm{dL}$ to $510 \mathrm{mg} / \mathrm{dL}$ until December 2009 (Fig. 3). Five months prior to that admission, the patient developed a productive cough without fever. At that time, his chest X-ray was normal and he was treated with a cough remedy and inhaled corticosteroids (ICS), which were ineffective for the symptoms. He had no additional past history, other than the fact that he had smoked one pack of cigarettes per day for 20 years and had stopped seven years earlier. He drank alcohol occasionally and did not use illicit drugs. As a policeman, he had no known occupational exposures and lived with his wife and daughter. The medication therapy administered upon admission included ICS.

A physical examination revealed a temperature of $36.9^{\circ} \mathrm{C}$, a blood pressure of 132/90, a pulse of 80 beats/min and an oxygen saturation of $91 \%$ while breathing ambient air. There were rhonchi in both lung fields and occasional wheezes. The remainder of the general physical and detailed neurologic examination was normal. The laboratory findings re- vealed a leukocyte count of 21,620 with $42 \%$ eosinophils. The patient's serum IgE level was $1,390 \mathrm{mg} / \mathrm{dL}$; however, his Aspergillus IgE radioallergosorbent test (RAST) score was negative. Tests for myeloperoxidase-antineutrophil cytoplasmic antibodies (MPO-ANCA), proteinase 3-antineutrophil cytoplasmic antibodies (PR3-ANCA) and precipitating antibodies against Aspergillus fumigatus were also negative. The serum level of KL-6, a marker of interstitial pneumonia, was $308 \mathrm{U} / \mathrm{mL}$ (normal range: $<499$ ) and that of surfactant protein (SP)-D was $110 \mathrm{ng} / \mathrm{mL}$ (normal range: < 110). Tests for urinary antigens for Streptococcus pneumoniae and Legionella were negative. A urinalysis revealed clear yellow urine with a $\mathrm{pH}$ of 6.5 , a specific gravity of 1.015 , no ketones, no blood, no protein and 0 to 1 white cells per high-power field. Cultures of blood, sputum and urine were sterile. Electrocardiogram revealed a sinus rhythm and was otherwise normal. It was not possible to complete a pulmonary function test (PFT) due to the patient's severe cough. Chest X-ray disclosed infiltrative shadows in both lung fields (Fig. 2a). A computed tomography (CT) scan of the chest performed without the administration of contrast material showed bilateral patchy infiltrations (Fig. 2b). To determine the cause of the lung infiltrates, bronchoalveolar lavage (BAL) was performed, which revealed a total cell count of $7.98 \times 10^{5} / \mathrm{mL}$ with $86 \%$ eosinophils; however, no acid-fast bacilli, fungus antigens or Pneumocystis jiroveci microorganisms were detected. A trans- 

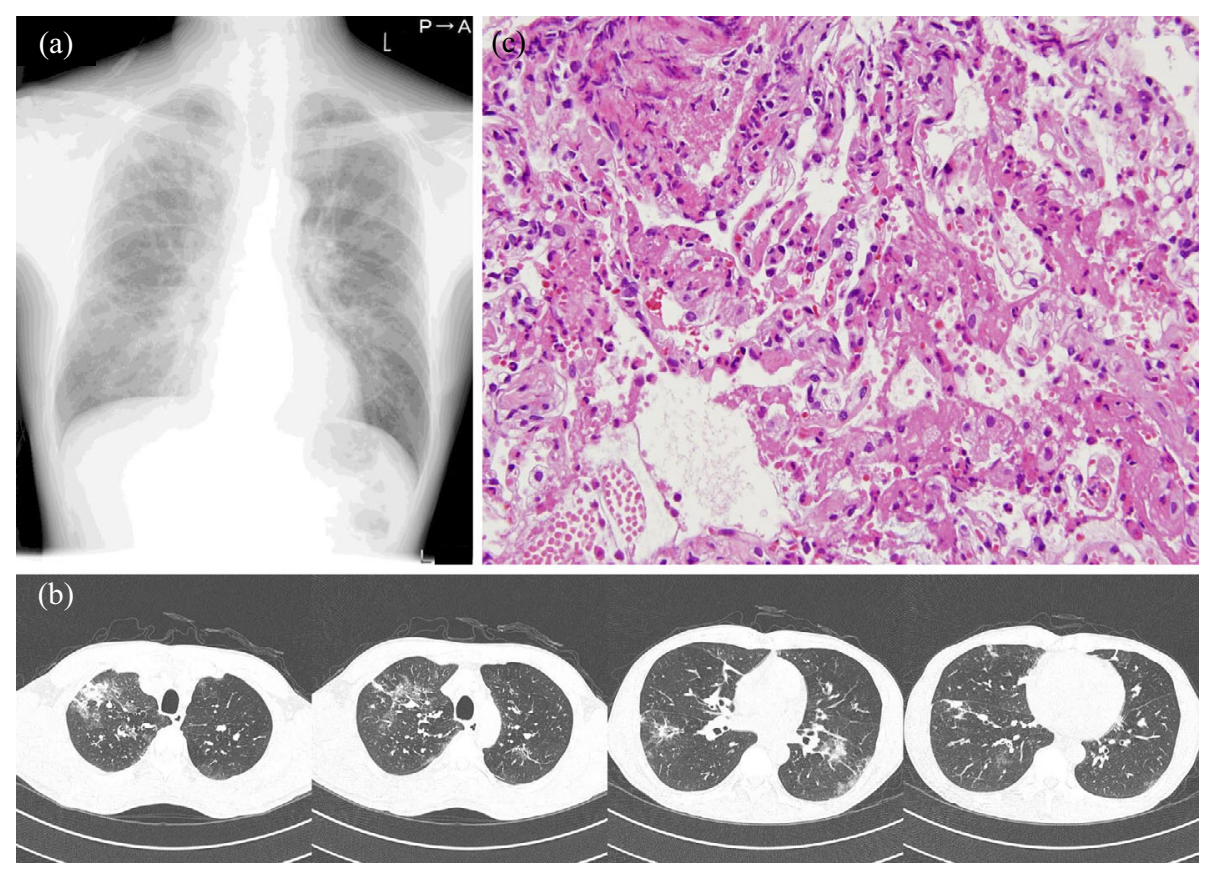

Figure 2. a: Chest X-ray obtained on admission demonstrating bilateral infiltrates. b: Chest CT scan obtained on admission showing bilateral patchy consolidation and ground glass opacities. c: A transbronchial biopsy specimen revealing eosinophil infiltration in the alveolar walls and airspaces $(\times 400)$.

bronchial lung biopsy specimen revealed eosinophil infiltration in the alveolar walls and airspaces without vasculitis, malignancy or granuloma (Fig. 2c). Based on these findings, a diagnosis of ELD was made. The clinical signs gradually resolved with steroid treatment, starting with $40 \mathrm{mg}$ of prednisone/day for two weeks, then gradually reducing the dose. The IgE level and eosinophil count both decreased following steroid therapy (Fig. 3).

Six months after the initiation of steroid therapy with the prednisone dose tapered to $2.5 \mathrm{mg} /$ day, the patient's clinical status worsened. He developed a productive cough and was subsequently re-admitted to our hospital. Chest X-ray and CT revealed bilateral patchy infiltrations. Blood and lung eosinophilia were detected, and a diagnosis of ELD recurrence was made. The patient's symptoms and infiltration improved following the administration of $30 \mathrm{mg}$ of prednisone. The dose was gradually reduced at the outpatient clinic, and the patient has continued to take $5 \mathrm{mg}$ of prednisone without recurrence of symptoms.

\section{Discussion}

$\mathrm{KD}$ is a rare, chronic inflammatory disorder of unknown etiology that occurs primarily in Asians and is characterized by benign slow-growing subcutaneous nodules $(1,2)$. It locates deep in subcutaneous tissue and in almost all cases involves the regional lymph nodes. However, the salivary glands, particularly the parotid, in addition to the oral cavity, axilla, groin and limbs, are occasionally involved. Histopathologically, lymphoid infiltrates with the formation of follicles and germinal centers accompanied by plasma cells, mast cells and particularly large amounts of eosinophils are present. Eosinophilic microabscesses and infiltration of germinal centers are also common (3).

Clinically, the differential diagnosis includes lymphoma, metastasis, Mikulicz's disease and angiolymphoid hyperplasia with eosinophilia (ALHE). KD and ALHE are similar in that both involve tissue swelling in the head and neck area. ALHE often affects middle-aged Western women and involves normal IgE levels. Histologically, both disorders are characterized by lymphoid hyperplasia, proliferation of blood vessels and eosinophil infiltration. However, the most important feature of ALHE is the proliferation of vessels lined by epithelioid endothelial cells, which is not observed in KD. Therefore, ALHE is regarded as a vascular tumor of epithelioid morphology and is also called epithelioid hemangioma, whereas $\mathrm{KD}$ is considered to be an immunemediated disorder (4-7).

Complications such as nephrotic syndrome (8-10), bronchial asthma $(11,12)$, atopic dermatitis, urticaria, ulcerative colitis (13) and aortitis syndrome (14) have been reported. In a review of 429 Japanese cases of KD, Ishii identified five cases associated with bronchial asthma, three cases associated with allergic rhinitis, two cases associated with atopic dermatitis and five cases associated with urticaria, suggesting that an allergic mechanism may contribute to KD (2). A high prevalence of nephropathy has been reported in patients with $\mathrm{KD}$ (8-10), and nephrotic syndrome is seen in the majority of reported cases, with most patients displaying mild histological changes on renal biopsies and many responding well to steroid therapy. The histological findings are mild to moderate, with membranous nephropathy, mini- 

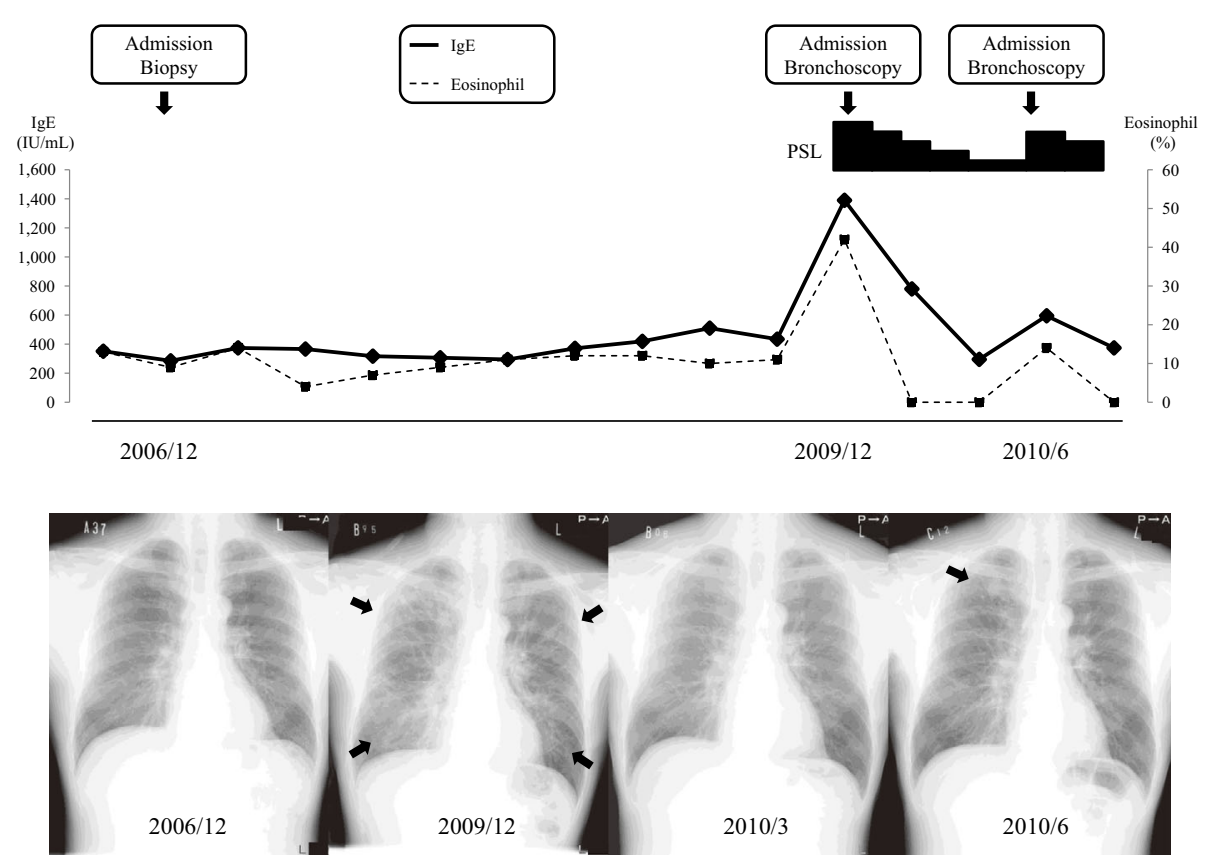

Figure 3. The patient's clinical course as indicated by chest X-ray and the levels of blood eosinophils and serum IgE. The arrows show lung infiltrations.

mal change nephrotic syndrome and mesangial proliferative glomerulonephritis having been reported to occur in association with the disorder. The pathogenesis of nephrotic syndrome in patients with KD may be regarded as multifactorial because previous reports have indicated many types of glomerulonephritis. Another speculation is that viral infections and toxins may stimulate the release of lymphokines by T-cell immunoregulation or the induction of IgEmediated type I hypersensitivity.

There are some case reports of bronchial asthma occurring in association with $\mathrm{KD}(11,12)$, such as those described by Tsukagoshi et al. (11). In their report, suplatast tosilate reduced the number of peripheral blood eosinophils and the serum levels of $\operatorname{IgE}$ and eosinophilic cationic proteins (ECPs), thereby improving asthma symptoms. However, in that report, suplatast tosilate did not affect the mRNA expression of TH1- or TH2-type cytokines in peripheral blood T-cells or the IL-5 mRNA expression in peripheral blood eosinophils. These results suggest that the mechanisms underlying KD and bronchial asthma differ with regard to antigen-specific and non-specific IgE production.

Tsubouchi et al. reported a case of IgG4-positive multi-organ lymphoproliferative syndrome associated with KD (15) in which chest CT revealed a tumor in the right lower lobe. The histological findings of a transbronchial biopsy showed inflammatory cells that consisted of plasma cells, lymphocytes and eosinophils. Immunohistochemical staining of the specimen showed infiltration of IgG4-positive plasma cells. The tumor disappeared after treatment with corticosteroids administered for KD.

Kitasato et al. described a case of KD accompanied by bilateral, mediastinal and hilar lymphadenopathy (16). The histological analysis of a cervical lymph node biopsy was compatible with a diagnosis of $\mathrm{KD}$. Although there have been reports of lung tumors, mediastinal lymphadenopathy and bronchial asthma occurring in relation to $\mathrm{KD}$, the presence of diffuse lung diseases such as ELD occurring in association with KD has not been described, with the exception of two unpublished case reports. One report described a pulmonary nodule complicated by KD. In that case, the cervical and pulmonary nodules were improved after treatment with corticosteroids; however, a pathological examination of the pulmonary nodule was not performed and no correct diagnosis was made. The case involved bronchial asthma and chronic eosinophilic pneumonia complicated by KD. No information was available beyond the title. Although unpublished reports include limited information, these cases and our report may suggest that KD and ELD share a common pathophysiology.

ELD includes simple pulmonary eosinophilia (Loffler's syndrome), acute eosinophilic pneumonia (AEP), chronic eosinophilic pneumonia (CEP), allergic granulomatosis and angitis (Churg-Strauss syndrome), hypereosinophilic syndrome, asthma, allergic bronchopulmonary aspergillosis (ABPA), bronchocentric granulomatosis, parasitic infections and drug and chemical reactions $(17,18)$. In our case, there was no evidence of parasitic infections or new drug reactions during the patient's clinical course. Additionally, there was no diagnosis of neuropathy, no paranasal or sinus abnormalities and no past history of bronchial asthma. Chest $\mathrm{X}$-ray and CT tests revealed bilateral infiltrates; however, the infiltrates were not peripherally predominant and no central bronchiectasis or pleural effusions were present. Furthermore, there were no symptoms of organ dysfunction. The most probable differential diagnosis included AEP and ABPA; however, the patient had only mild hypoxemia and 
no typical asthmatic symptoms. A test for precipitating antibodies against Aspergillus fumigatus was negative. Expectoration of mucous plugs was not detected on bronchoscopy. Consequently, making a definite diagnosis was difficult. Eventually, the patient was diagnosed as having ELD based on laboratory findings, BAL fluid results and a biopsy specimen. His condition responded completely to corticosteroids; however, it worsened after the dose was tapered. PFT was not performed due to the patient's severe cough, and TBLB was not detected in the bronchial lesion in this case. However, on the basis of the lesion presenting in association with bronchovascular bundles and wheezing on a physical examination, it is possible that the main focus of ELD in this case included not only the alveolar space, but also the bronchial lesion.

Eosinophils play a role in many inflammatory processes occurring in a variety of organs. ELD is primarily represented by eosinophilic infiltration of the lung parenchyma or airways. Hence, ELD corresponds to a wide and heterogeneous spectrum of disorders and may manifest clinically as pulmonary diseases of varying severity, ranging from chronic or transient infiltrates with mild symptoms to acute severe eosinophilic pneumonia. It is unclear whether the pathophysiology of $\mathrm{KD}$ is different from that of ELD, as this is a particularly complicated issue. Further research is needed to clarify the association. To the best of our knowledge, this is the first report of ELD complicated by KD.

The authors state that they have no Conflict of Interest (COI).

\section{References}

1. Kimura T, Yoshimura S, Ishikawa E. Unusual granulation combined with hyperplastic change of lymphatic tissue. Trans Soc Pathol Jpn 37: 179-180, 1948.

2. Ishii M. Kimura's disease: a review of 429 cases and four new cases. Oto-Rhino-Laryngology Tokyo 25: 407-416, 1982.

3. Ioachim HL, Medeiros LJ. Kimura lymphadenopathy. In: Ioachim's Lymph Node Pathology. 4th ed. Ioachim HL, Medeiros LJ, Eds. Lippincott Williams \& Wilkins, Philadelphia, 2008: 190192.

4. Saxe N, Kahn LB. Angiolymphoid hyperplasia with eosinophilia.
Report of 3 cases. S Afr Med J 52: 454-457, 1977.

5. Sheren SB, Custer PL, Smith ME. Angiolymphoid hyperplasia with eosinophilia of the orbit associated with obstructive airway disease. Am J Ophthalmol 108: 167-169, 1989.

6. Dupre A, Viraben R. Coexistence of angiolymphoid hyperplasia with eosinophilia and pulmonary neoplasia. J Am Acad Dermtol 16: 142-144, 1987.

7. Moran CA, Suster S. Angiolymphoid hyperplasia with eosinophilia (Epithelioid hemangioma) of the lung. A clinicopathologic and immunohistochemical study. Am J Clin Pathol 123: 762-765, 2005.

8. Whelan TV, Maher JF, Kragel P, et al. Nephrotic syndrome associated with Kimura's disease. Am J Kidney Dis 11: 353-356, 1988.

9. Matsumoto K, Katayama H, Hatano M. Minimal-change nephritic syndrome associated with subcutaneous eosinophilic lymphoid granuloma (Kimura's disease). Nephron 49: 251-254, 1988.

10. Obata Y, Furusu A, Nishino T, et al. Membranous nephropathy and Kimura's disease manifesting a hip mass. A case report with literature review. Intern Med 49: 1405-1409, 2010.

11. Tsukagoshi H, Nagashima M, Horie T, et al. Kimura's disease associated with bronchial asthma presenting eosinophilia and Hyperimmunoglobulinemia $\mathrm{E}$ which were attenuated by suplatast tosilate (IPD-1151T). Intern Med 37: 1064-1067, 1998.

12. Ito S, Kume H, Kimura T, et al. Two cases of Kimura's disease associated with bronchial asthma. Nihon Kokyuki Gakkai Zasshi 37: 1008-1012, 1999.

13. Sugaya M, Suzuki T, Asahina A, et al. Kimura's disease associated with ulcerative colitis: detection of IL-5 mRNA expression of peripheral blood mononuclear cells and colon lesion. Acta Derm Venereol 78: 375-377, 1998.

14. Horie $\mathrm{S}$, Ishiyama $\mathrm{T}$, Sugimoto $\mathrm{M}$, et al. Eosinophilic lymphfolliculosis (Kimura's disease) complicated with aortitis syndrome. Jpn J Clin Hematol 30: 396-399, 1989.

15. Tsubouchi K, Imanaga $T$, Yamamoto $M$, et al. A case of IgG4positive multi-organ lymphoproliferative syndrome associated with Kimura's disease. Nihon Kokyuki Gakkai Zasshi 48: 524-528, 2010.

16. Kitasato Y, Tajiri M, Matsunaga K, et al. A case of Kimura's disease accompanied with bilateral, mediastinal and hilar lymphadenopathy. Nihon Kokyuki Gakkai Zasshi 43: 252-257, 2005.

17. Cordier JF, Cottin V. Eosinophilic lung disease. In: Murray's and Nadel's Textbook of Respiratory Medicine. 4th ed. Mason RJ, Broaddus VC, Murray JF, Nadel JA, Eds. Elsevier Saunders, Philadelphia, 2005: 1679-1701.

18. Cordier JF. Eosinophilic pneumonia. In: Interstitial Lung Disease. 4th ed. Schwarz MI, King TE Jr, Eds. BC Decker, Hamilton, 2003: 657-700.

(C) 2012 The Japanese Society of Internal Medicine http://www.naika.or.jp/imonline/index.html 